\title{
Critical Behavior of Nuclear-Spin Diffusion in GaAs/AlGaAs Heterostructures near Landau Level Filling $\nu=1$
}

\author{
V. Bayot ${ }^{1}$, E. Grivei ${ }^{1}$, J.-M. Beuken ${ }^{1}$, S. Melinte ${ }^{1}$ and M. Shayegan ${ }^{2}$ \\ ${ }^{1}$ Unité de Physico-Chimie et de Physique des Matériaux, Université Catholique de Louvain, \\ Place Croix du Sud 1, 1348 Louvain-la-Neuve, Belgium \\ ${ }^{2}$ Department of Electrical Engineering, Princeton University, Princeton N.J. 08544
}

(March 30, 2018)

\begin{abstract}
Thermal measurements on a GaAs/AlGaAs heterostructure reveal that the state of the confined two-dimensional electrons dramatically affects the nuclear-spin diffusion near Landau level filling factor $\nu=1$. The experiments provide quantitative evidence that the sharp peak in the temperature dependence of heat capacity near $\nu=1$ is due to an enhanced nuclear-spin diffusion from the GaAs quantum wells into the AlGaAs barriers. We discuss the physical origin of this enhancement in terms the possible Skyrme solid-liquid phase transition.
\end{abstract}

PACS numbers: 73.20.Dx, 73.40.Hm, 65.40.+g 
In the presence of a perpendicular magnetic field $(B)$, two-dimensional electron systems (2DESs) exhibit striking phenomena originating from the Landau quantization and electronelectron interaction; examples are the integral and fractional quantum Hall effects [1]. At Landau level filling factor $\nu=1$, theoretical work on 2DESs in GaAs/AlGaAs heterostructures has shown that electron spin textures known as Skyrmions are the lowest energy, charged excitations of the ferromagnetic ground state [2]. Skyrmions, which result from the dominance of the electron-electron exchange energy over the Zeeman energy, are also the lowest energy state for quasi-holes and quasi-electrons near $\nu=1$; the ground state of a 2DES near $\nu=1$ is in fact expected to be a crystal of Skyrmions [3 5 .

Recent experimental studies have revealed that Skyrmions play a major role in many properties of quantum Hall systems [6 9]. In particular, measurements on a GaAs/AlGaAs multiple-quantum-well (QW) heterostructure indicated an anomalous behavior for the lowtemperature apparent-heat-capacity $(C)$ near $\nu=1$ : $C$ is enhanced by a factor of up to $\sim 10^{5}$ with respect to its low- $B$ value [9]. For temperatures $T \gtrsim 0.1 \mathrm{~K}$, the magnitude as well as $T$ - and $B$-dependence of the data are consistent with the large $C$ being dominated by the Schottky nuclear heat capacity of Ga and As atoms in the QWs. This observation implies a strong enhancement of the nuclear spin-lattice relaxation rate near $\nu=1$, consistent with the results of nuclear magnetic resonance (NMR) experiments [6,7] and recent calculations [5] which attribute the enhancement to the presence of low-energy spin-flip excitations in the 2DES. An even more striking feature of the $C$ vs $T$ data is a very sharp peak at a very low temperature $T_{c}(\nu)$, suggestive of a phase transition in the 2DES [9]. While recent estimates for the melting temperature of the Skyrme crystal [ [. [- including its dependence on $\nu$, suggest that the peak in $C$ could originate from a Skyrme solid-liquid phase transition, the physical mechanism that affects the nuclear spin dynamics and gives rise to the anomalous $C$-peak is not known and is a matter of debate [4, 5 ,9].

In this Letter we report new quasi-adiabatic thermal experiments revealing that the mechanism responsible for the peak in $C$ vs $T$ is a dramatic enhancement of nuclear spin diffusion across the QW-barrier interface. While only the nuclear heat capacity of the QWs' 
atoms is observed away from $T_{c}$, the nuclei in both the QWs and barriers contribute to the measured heat capacity near $T_{c}$. We discuss the physical origin of this phenomenon in terms of the possible Skyrme solid-liquid phase transition.

The experiments were performed on the same multiple-QW heterostructure used in Ref. [9]. It consists of 100 GaAs QWs separated by $\mathrm{Al}_{0.3} \mathrm{Ga}_{0.7} \mathrm{As}$ barriers. The wells and barriers are 250 and $1850 \AA$ thick, respectively, and the barriers are $\delta$ doped with donors ( $\mathrm{Si}$ ) near their centers. We used a $7 \times 7 \mathrm{~mm}^{2}$ piece of the wafer which was thinned to $65 \mu \mathrm{m}$. The lattice $T$ is probed by a carbon paint resistor deposited on the substrate side of the sample and electrically connected with four very low thermal conductance, $8 \mu \mathrm{m}$-diameter, NbTi wires. This resistor was calibrated against a $\mathrm{RuO}_{2}$ resistance thermometer at $B=0$. Since the nuclear spin temperature $\left(T_{N}\right)$ may be inhomogeneous in the sample due to a variable nuclear spin-lattice relaxation rate [6], $T$ strictly refers to the measured lattice temperature. The sample is supported by two $60 \mu \mathrm{m}$-diameter NbTi wire which serves as the main weak thermal link to the cold finger of the dilution refrigerator mixing chamber. In the restricted $T$ and $\nu$-range investigated here, $C$ is very large and the observed external time constant, $\tau_{\text {ext }}$, reaches extremely large values which exceed $10^{4}$ seconds. Therefore, in the time scale of our experiments, which is governed by the observed internal time constant $\tau_{\text {int }} \sim 10^{3} \mathrm{~s}$ (see below), the sample is in the quasi-adiabatic regime. $B$ is applied at an angle of $\theta=30^{\circ}$ with respect to the 2DES plane in order to match the experimental conditions of Ref. [9].

We first summarize the heat capacity results of Ref. [9]. Figure 1] shows the measured $C$ vs $T$ at $\nu=0.77$, revealing a sharp peak at $T_{c}=42 \mathrm{mK}$. While at high $T(\gtrsim 70 \mathrm{mK})$ the measured value and $T$-dependence of $C$ are rather consistent with the calculated Schottky nuclear heat capacity of Ga and As atoms in the QWs [9]10] (solid curve in Fig. 1), at lower $T$, and especially near $T_{c}, C$ exceeds the calculated value by a factor of up to $\sim 10$. The peak value of $C$, however, appears consistent with the Schottky nuclear heat capacity of the heterostructure if the nuclei of the barrier atoms are also included (dotted curve in Fig. (1)). This observation suggests that, near $T_{c}$, the barriers' nuclear spins also contribute to the measured $C$. 
In our new quasi-adiabatic experiments, we utilize another noteworthy feature of the

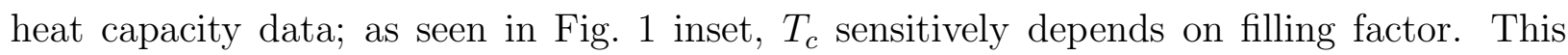
dependence, which is reproduced in Fig. 2 (a) (dashed curve), is the key to understanding our results. In these experiments, we first fix the magnetic field at $B=8.5 \mathrm{~T}(\nu=0.77)$ where the peak in $C$ occurs at $T_{c}=42 \mathrm{mK}$ (Fig. [1 and dashed curve in Fig. 2(a)). Starting from $T \sim 60 \mathrm{mK}$, we cool down the cold finger to base temperature $(\sim 10 \mathrm{mK})$ and the lattice slowly cools down to $T \sim 32 \mathrm{mK}$ by waiting for about 50 hours. Then, in order to further improve the adiabatic conditions, the weak heat flow that results from the $T$-difference between the lattice and the cold finger is reduced by increasing the cold finger temperature to $32 \pm 3 \mathrm{mK}$. Under these conditions, we observe that the measured sample $T$ is stable within the experimental accuracy ( $\lesssim \pm 0.2 \mathrm{mK}$ ) over periods longer than $\tau_{\text {ext }}$, meaning that the sample is effectively quasi-adiabatic.

In a first experiment, whose results are shown by open circles in Fig. Q(a), we started from such a quasi-adiabatic condition and swept $B$ from 8.5 to $7.7 \mathrm{~T}$, at a rate of $0.01 \mathrm{~T} /$ minute. We observe that $T$ rises from $31.7 \mathrm{mK}$ to $36.2 \mathrm{mK}$. $B$ was then swept back to $8.5 \mathrm{~T}$ at the same rate; this led to a rise of $T$ to $39.5 \mathrm{mK}$. While the increase in $T$ with increasing $B$ may be explained by the adiabatic magnetization of the nuclei in the QWs, which couple to the lattice, according to [10]:

$$
T_{N} \propto B
$$

the $T$ rise observed when $B$ is lowered from $8.5 \mathrm{~T}$ to $7.7 \mathrm{~T}$ is puzzling.

In order to better understand the results of the above experiment, we performed a similar experiment, starting from $T=32.6 \mathrm{mK}$, but here we swept $B$ in steps of $0.2 \mathrm{~T}$ from $8.5 \mathrm{~T}$ to 7.3T, with a hold time of 3 hours between steps. The evolution of $T$ during this second experiment is presented in Fig. 2(b) and summarized in Fig. 2(a) by close circles. We note that in each step, except for steps $2 \rightarrow 3$ and $3 \rightarrow 4$, the lattice $T$ rises during the $B$-sweeps and then decays to approximately the same value as the one at the end of the previous step. These $T$ rises are due to Eddy-current heating of the 2DES and the lattice during 
the $B$-sweeps. The heat is evidently slowly absorbed by the nuclei during and following the sweep via internal relaxation. Because of the very large heat capacity of the nuclear spins, this (Eddy-current) heating does not lead to an appreciable increase in the lattice $T$ at the end of the step [11]. The key feature of this experiment, however, is that during the $2 \rightarrow 3$ and $3 \rightarrow 4$ steps, the lattice $T$ increases significantly while the sample is in quasi-adiabatic conditions. Note in particular that, during the $2 \rightarrow 3$ step, the lattice $T$ rises even after the $B$-sweep is completed. This striking behavior occurs when the lattice $T$ approaches $T_{c}$ at which $C$ exhibits a maximum (dashed curve in Fig. 2(a)). Indeed, it appears that near $T_{c}$ the lattice is heated internally.

To explain the results of Figs. 11 and 2, we propose a model in which the barriers' nuclei couple to the QWs' nuclei very near $T_{c}$, but decouple at higher and lower temperatures. The sharpness of the peak in $C$ vs $T$ data (Fig. 1) is consistent with this model and implies that the coupling between the QWs and barriers is turned off rapidly as $T$ deviates from $T_{c}$. Therefore, we expect that during the initial cool-down at $B=8.5 \mathrm{~T}$, when $T$ decreases below $T_{c}=42 \mathrm{mK}, T_{N}$ in the AlGaAs barriers remains close to $42 \mathrm{mK}$ while $T_{N}$ of the QWs decreases down to $\approx 32 \mathrm{mK}$ due to strong coupling to the lattice. Next, decreasing $B$ below 8.5T (steps $1 \rightarrow 7$ ) in adiabatic conditions has two distinct consequences: (1) $T_{N}$ in both the QWs and barriers is reduced due to adiabatic demagnetization of the nuclei (Eq. 1), and (2) the coupling between the QWs and the barriers will increase dramatically when the dashed ( $T_{c}$ vs $\left.B\right)$ curve in Fig. 2(a) is crossed. While the former is essentially monotonic in $B$, the latter is not and implies that, near the dashed curve in Fig. 2(a), $T_{N}$ should equalize over the whole GaAs/AlGaAs heterostructure. Since the barriers' nuclei were initially warmer than the QWs' nuclei, we expect a rise in lattice $T$ near this crossing, as observed experimentally. Finally, we attribute the $T$ rise during the final $(7 \rightarrow 8)$ step to the nuclear magnetization of the nuclei (Eq. 四).

Beyond the good qualitative description of the experiments in Fig. Q, the above model appears to provide a reasonable quantitative account of the data also. First, when $B$ is lowered from $8.5 \mathrm{~T}$ to $7.3 \mathrm{~T}$, we expect from Eq. 1 that nuclear demagnetization reduces $T_{N}$ 
in the barriers from $\approx 42 \mathrm{mK}$ to $\approx 36 \mathrm{mK}$. Since the barriers are by far thicker than the QWs, according to our model, this value should approximately correspond to the temperature of the entire heterostructure, and hence of the lattice. This prediction is in good agreement with experiments that give $T \approx 37 \mathrm{mK}$ at $\mathrm{B}=7.3 \mathrm{~T}$. The final increase of $T$ from $\approx 37 \mathrm{mK}$ to $\approx 43 \mathrm{mK}$ when $B$ increases from $7.3 \mathrm{~T}$ to $8.5 \mathrm{~T}$ is consistent with the nuclear magnetization of the heterostructure (Eq. 11). Second, the fact that, at $T_{c}, C$ reaches the value expected for the entire heterostructure (dotted curve in Fig. 1) is clearly consistent with our model and hence provides additional credence to our interpretation.

Next, we discuss the internal time constant $\left(\tau_{\text {int }}\right)$ observed in our experiments. Figure 3 presents a typical heat pulse/relaxation trace, in quasi-adiabatic conditions, obtained during heat capacity experiments near $T_{c}$. The relaxation follows an exponential decay characterized by $\tau_{\text {int }}$. The inset to Fig. 3 shows that $\tau_{\text {int }}$ exhibits two different, but remarkably constant, values above and below 30mK: $\sim 1600$ s and $\sim 900$ s, respectively. We note that this crossover temperature corresponds to the $T$ below which $C$ is reduced back to a value close to the nuclear heat capacity of the 100-QWs, $C_{Q W}$ (full curve in Fig. 1).

In order to explain these observations, we consider three possible mechanisms responsible for the observed $\tau_{\text {int }}$. Heat diffusion in the nuclear spin system of the barriers is governed by the one-dimensional diffusion equation:

$$
\frac{\partial T}{\partial t}=D \frac{\partial^{2} T}{\partial z^{2}}
$$

where $D \sim 10^{-17} \mathrm{~m}^{2} / \mathrm{s}$ is the nuclear-spin diffusion constant in GaAs [12] and the $z$-axis is along the growth direction. Therefore, it gives an internal time constant:

$$
\tau_{d} \sim \frac{r^{2}}{D}
$$

where $r$ is the distance over which diffusion takes place [13]. Since each barrier is surrounded by two QWs, we take $r=925 \AA$ (half the barrier thickness) and Eq. 3 gives $\tau_{d} \sim 800 \mathrm{~s}$. The two other mechanisms involved in the heat transfer from the lattice to the barrier nuclei, i.e. nuclear spin-lattice relaxation in the QWs and diffusion across the QW-barrier interfaces, result in two additional time constants: $T_{1}$ and $\tau_{i}$, respectively. When $C \approx C_{Q W}(T \lesssim 30 \mathrm{mK})$, 
the diffusion across the QW-barrier interface is negligible and $\tau_{\text {int }}$ should be determined by $T_{1}$ only. On the other hand, when $C>C_{Q W}(30 \lesssim T \lesssim 50 \mathrm{mK})$ at least a fraction of the barriers' nuclei couple to the QWs' nuclei. This implies that $\tau_{d}$ and $\tau_{i}$ should increase $\tau_{\text {int }}$. This is consistent with the rise in $\tau_{\text {int }}$ observed above $30 \mathrm{mK}$ (Fig. 3 inset). Moreover, we note that the increase of $\tau_{\text {int }}$ above $30 \mathrm{mK}(\sim 700 \mathrm{~s})$ is comparable to the estimated $\tau_{d} \sim 800 \mathrm{~s}$, implying that $\tau_{i}$ is rather small once the barrier nuclei do couple to those in the QWs [14].

We now remark on the physical origin of the peak in $C$ vs $T$. NMR experiments show that nuclear spin-diffusion from the QWs into the barriers is extremely weak, except when optical pumping broadens the Knight shift peak of the QWs which then overlaps with the NMR peak of the barriers [6]. The spectral overlap allows spin-diffusion which is driven by nuclear magnetic dipole-dipole coupling [6,15]. Therefore, the enhancement of spin-diffusion across the QW-barrier interface near $T_{c}$ could originate from a broadening of the Knight shift peak in the QWs. In the liquid Skyrme phase, motional averaging of the local 2DES spin polarization produces a single Knight shift peak [3,7]. On the other hand, the absence of motional averaging in the solid Skyrme phase should induce both positive and negative Knight shifts depending on the local spin polarization of the 2DES. Since above and below the peak in $C$ vs $T$ the diffusion across the QW-barrier interface is very weak, this implies that in both the liquid and the solid Skyrme states, the Knight shift peak(s) do not overlap with the NMR peak of the barrier. One possible explanation for the $C$ anomaly is that the critical slowing down of the spin fluctuations in the 2DES, associated with the Skyrme liquid-solid phase transition [5], could induce a broadening of the Knight shift peak in the QWs. This broadening would induce spectral overlap of the QWs' and barriers' NMR peaks and hence allow spin-diffusion across the QW-barrier interface only near $T_{c}$.

Finally, it is worth emphasizing that the shape of the peak in $C$ vs $T$ points to a secondorder phase transition [16]. The fact that $\tau_{\text {int }}$ is constant in the $T$-range where $C>C_{Q W}$ near $T_{c}$ implies that the strength of the coupling to the barriers remains constant while $C$ varies strongly. Therefore, it appears that the smaller value of $C$ on the sides of the peak in $C$ vs $T$ comes from a reduced fraction of the sample in which the barriers' nuclei couple to 
the QWs' nuclei. The complexity of spin textures in the 2DES, together with the fact that the phase transition is not observed directly but through its effect on nuclear-spin lattice relaxation, does not allow a quantitative analysis of the data in the framework of the existing theories that describe order-disorder phase transitions. On the other hand, Skyrmions form an XY ferromagnet which has broken translation symmetry and one expects at least one phase transition as $T$ is lowered [5].

In conclusion, our experiments reveal the critical influence of the ground and excited states of the 2DES near $\nu=1$ on nuclear-spin dynamics in GaAs/AlGaAs heterostructures. Our results provide a quantitative phenomenological description of the apparent heat capacity anomaly that may originate from a Skyrme solid-liquid phase transition.

The authors are much indebted to S.E. Barrett, S.M. Girvin and A.H. MacDonald for fruitful discussions and suggestions. This work has been supported by NATO grant CRG 950328 and the NSF MRSEC grant DMR-9400362. V.B. acknowledges financial support of the Belgian National Fund for Scientific Research. 


\section{REFERENCES}

[1] For a review of the QHE see "The Quantum Hall Effect", edited by R.E. Prange and S.M. Girvin (Springer-Verlag, New York, 1987) and "Perspectives in Quantum Hall Effects", edited by S. Das Sarma and A. Pinczuk (Wileys, New York, 1997).

[2] D.-H. Lee and C.L. Kane, Phys. Rev. Lett. 64, 1313 (1990); S.L. Sondhi, A. Karlhede, S.A. Kivelson and E.H. Rezayi, Phys. Rev. B 47, 16419 (1993); H.A. Fertig, L. Brey, R. Côté and A.H. MacDonald, Phys. Rev. B 50, 11018 (1994); N. Read and S. Sachdev, Phys. Rev. Lett. 75, 3509 (1995); X.-G. Wu and S.L. Sondhi, Phys. Rev. B 51, 14725 (1995).

[3] L. Brey, H.A. Fertig, R. Côté and A.H. MacDonald, Phys. Rev. Lett. 75, 2562 (1995).

[4] A.G. Green, I.I. Kogan and A.M. Tsvelik, Phys. Rev. B 54, 16838 (1996).

[5] R. Côté, A.H. MacDonald, L. Brey, H.A. Fertig, S.M. Girvin and H.T.C. Stoof, LANL cond-mat preprint 970220 (1997).

[6] R. Tycko, S.E. Barrett, G. Dabbagh, L.N. Pfeiffer and K.W. West, Science 268, 1460 (1995).

[7] S.E. Barrett, G. Dabbagh, L.N. Pfeiffer, K.W. West and R. Tycko, Phys. Rev. Lett. 74, $5112(1995)$.

[8] A. Schmeller, J.P. Eisenstein, L.N. Pfeiffer and K.W. West, Phys. Rev. Lett. 75, 4290 (1995); E.H. Aifer, B.B. Goldberg and D.A. Broido, Phys. Rev. Lett. 76, 680 (1996); D.K. Maude et al., Phys. Rev. Lett. 77, 4604 (1996).

[9] V. Bayot, E. Grivei, S. Melinte, M.B. Santos and M. Shayegan, Phys. Rev. Lett. 76, 4584 (1996).

[10] for a review see e.g. H.M. Rosenberg, Low Temperature Solid State Physics (Clarendon Press, Oxford, 1963). 
[11] Complementary experiments allow us to estimate the $T$ increase expected from the Eddy-current heating during the $B$-sweeps. Our estimate is $\lesssim 2 \mathrm{mK}$ for the total $T$ rise during steps 1 to 8 . This may explain the slightly larger $T(\approx 43 \mathrm{mK})$ measured at the end of step 8 compared to the starting barrier $T_{N}$ which is expected to be $\approx 42 \mathrm{mK}$ according to our model.

[12] D. Paget, Phys. Rev. B 25, 4444 (1982). In our estimate here, we use D for GaAs since $D$ for $\mathrm{Al}_{0.3} \mathrm{Ga}_{0.7} \mathrm{As}$ is not known.

[13] See e.g. R.P. Tye, Thermal conductivity, (Academic Press, London, 1969) p. 168. Note that the actual boundary conditions used to solve Eq. 2 may add a prefactor to the right term of Eq. 3 which gives only a rough estimation of $\tau_{d}$.

[14] It is worth remarking that the parts of the sample for which $\tau_{d} \gg \tau_{\text {ext }}$ do not contribute to the measured $C$ in the $T$ and $B$ range we are discussing. Since $\tau_{d} \gtrsim 10^{5}$ s for $r>10^{-6}$ m (Eq. 3), the GaAs substrate contributes negligibly to $C$.

[15] D. Suter and R.R. Ernst, Phys. Rev. B 32, 5608 (1985); R. Tycko and G. Dabbagh, Isr. J. Chem. 32, 179 (1992).

[16] See e.g. J.M. Ziman in "Principles of the Theory of Solids", (Cambridge University Press, Cambridge, 1972), p. 353. 


\section{FIGURES}

FIG. 1. Measured $C$ vs $T$ at $B=8.5 \mathrm{~T}$ and $\theta=30^{\circ}(\nu=0.77)$. The curves represent the calculated Schottky nuclear heat capacity of the GaAs 100-QWs (full curve) and the 100-period GaAs $/ \mathrm{Al}_{0.3} \mathrm{Ga}_{0.7} \mathrm{As}$ heterostructure (dotted curve). The inset shows the measured $\nu$-dependence of $T_{c}$ at $\theta=30^{\circ}$.

FIG. 2. Measured lattice $T$ during the quasi-adiabatic experiments. (a) summarizes the evolution of $T$ during the first (o) and the second $(\bullet)$ experiments (see text). The full and the dotted lines are guides to the eye and the arrows indicate the direction of evolution. The dashed curve corresponds to the $B$-dependence of $T_{c}$ as reproduced from Fig. 1 inset. (b) shows $T$ and $B$ vs

time in the second experiment. $B$-sweeps (gray areas) separate hold times at the end of which $T$ is recorded ( 1 to 7 ) and summarized in (a) together with the final $T$ at $B=8.5 \mathrm{~T}$ (8).

FIG. 3. $T$ vs time during a heat capacity experiment in the quasi-adiabatic regime $(C=Q / \Delta T$ where $Q$ is the heat injected during the pulse; $B=8.5 \mathrm{~T}$ and $\theta=30^{\circ}$ ). The heat pulse is followed by a relaxation characterized by an exponential decay (full curve) giving $\tau_{\text {int }} \approx 1500$ s. The inset shows $\tau_{\text {int }}$ vs $T$ at the same $B$ and $\theta$. 


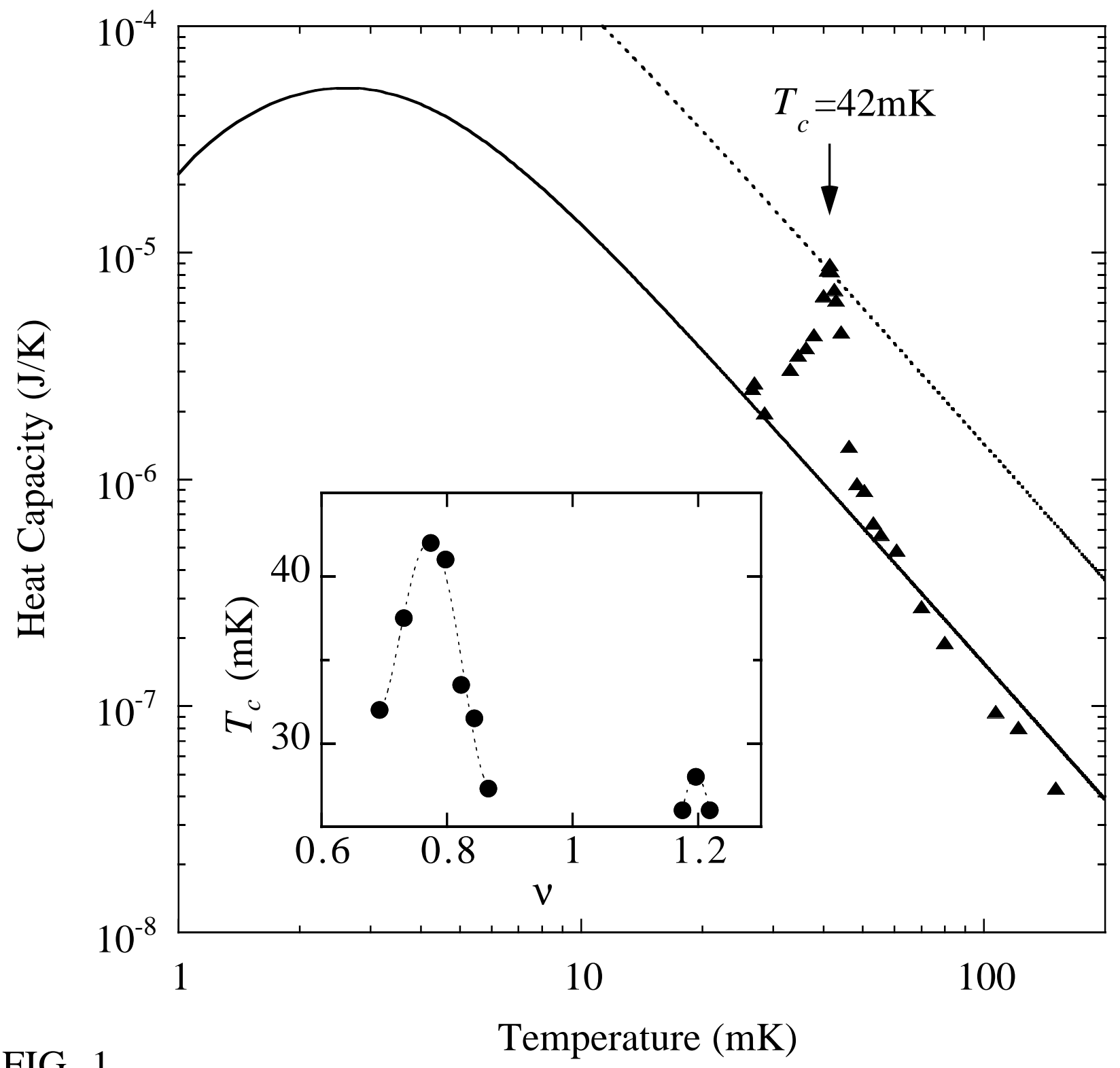

FIG. 1 

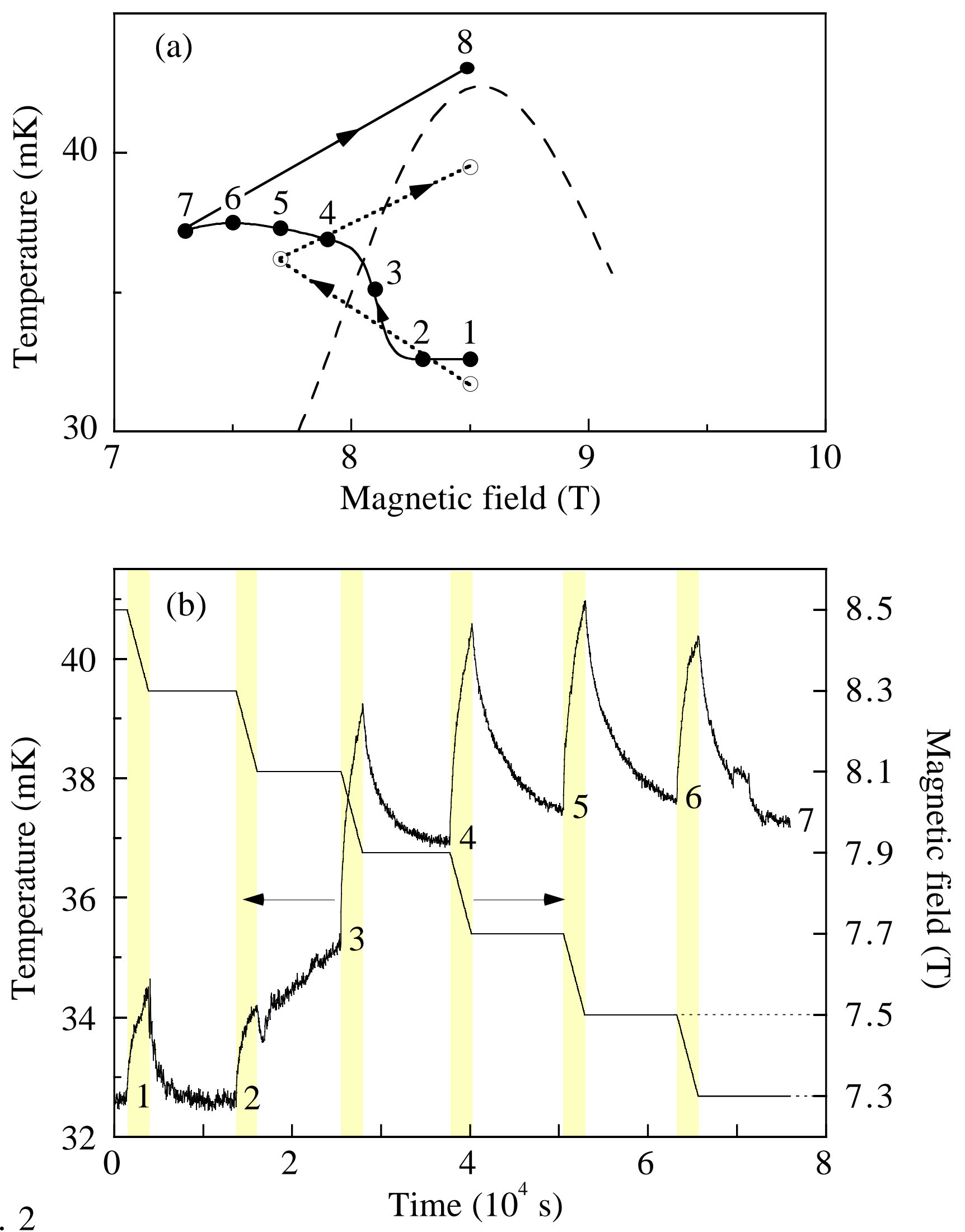

FIG. 2 


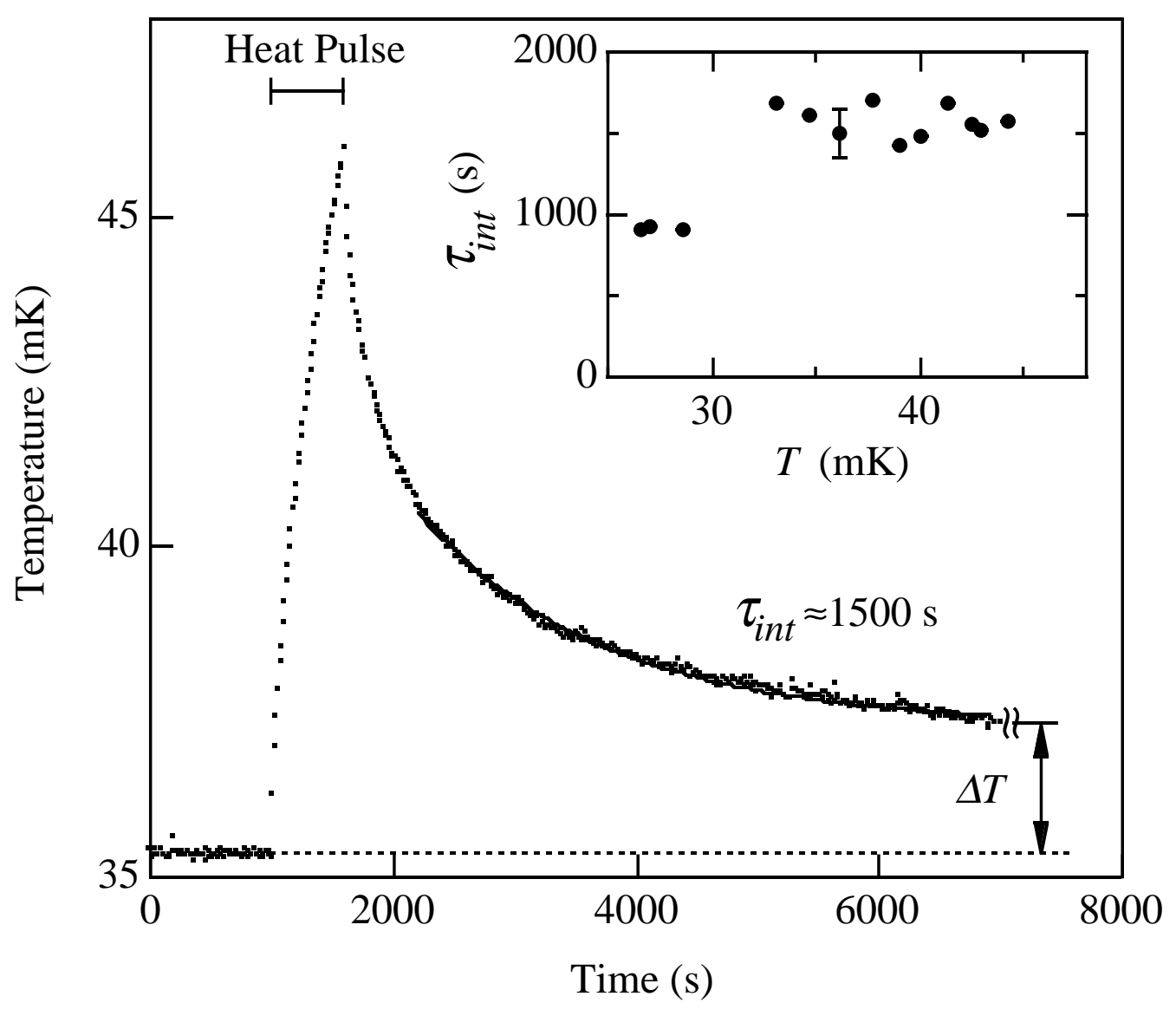

FIG. 3 